\title{
Alteration of Cortical Functional Networks in Default-Mode Network-Related Regions in Mood Disorders with Resting-State Electroencephalography
}

\section{Sungkean Kim}

University of Florida

Ji Hyun Baek

Samsung Medical Center

Sehoon Shim

Soon Chun Hyang University Cheonan Hospital

\section{Young Joon Kwon}

Soon Chun Hyang University Cheonan Hospital

Hwa Young Lee

Soon Chun Hyang University Cheonan Hospital Jae Hyun Yoo

Catholic University of Korea

Ji Sun Kim ( $\square$ ideal91@hanmail.net)

Soon Chun Hyang University Cheonan Hospital

\section{Research Article}

Keywords: bipolar disorder, cortical functional network, graph theory, major depressive disorder, restingstate electroencephalography

Posted Date: December 10th, 2020

DOI: https://doi.org/10.21203/rs.3.rs-119899/v1

License: (c) (i) This work is licensed under a Creative Commons Attribution 4.0 International License. Read Full License 


\section{Abstract}

Studies comparing bipolar disorder (BD) and major depressive disorder (MDD) are scarce, and the neuropathology of these disorders is poorly understood. This study investigated source-level cortical functional networks using resting-state electroencephalography (EEG) in patients with BD and MDD. EEG was recorded in 35 patients with BD, 39 patients with MDD, and 42 healthy controls (HCs). Graph theorybased source-level weighted functional networks were assessed via strength, clustering coefficient (CC), and path length $(\mathrm{PL})$ in six frequency bands. At the global level, patients with BD and MDD showed higher strength and $\mathrm{CC}$, and lower PL in the high beta band, compared to HCs. At the nodal level, compared to HCs, patients with BD showed higher high beta band nodal CCs in the right precuneus, left isthmus cingulate, bilateral paracentral, and left superior frontal, belonging to the default-mode network (DMN); however, patients with MDD showed higher nodal CC only in the right precuneus compared to HCs. Although both MDD and BD patients had similar global level network changes, they had different nodal level network changes in DMN-related regions. Our findings might suggest more altered network in the DMN-related regions in patients with BD than in those with MDD.

\section{Introduction}

Bipolar disorder (BD) and major depressive disorder (MDD) are considered representative mood disorders. These two psychiatric conditions exhibit similar severe depressive symptoms and show no difference in the duration of affective episodes during the course of illness ${ }^{1}$. Since misdiagnosis of BD as MDD is a serious clinical problem ${ }^{2}$, differential diagnoses of BD and MDD is paramount for clinicians to avoid risks of misdiagnosis and administration of inappropriate medication regimen ${ }^{1}$. Therefore, promising biomarkers are needed to support the differential diagnosis between the two conditions.

Previous neuroimaging studies demonstrated widespread brain structural and functional alterations in both BD and MDD, such as the prefrontal cortex, limbic system, ventral striatum, and thalamus ${ }^{3-6}$. However, studies on direct comparisons between BD and MDD are sparse, with inconclusive results. Structural studies reported more widespread abnormalities in white matter connectivity and hyperintensities in $\mathrm{BD}$ than in MDD ${ }^{3,7}$. One functional magnetic resonance imaging (fMRI) study ${ }^{8}$ found that cortical functional connectivity in the right posterior cingulate could distinguish between BD and MDD. However, other fMRI studies 9,10 failed to observe significant differences in functional connectivity between the two disorders.

Both patients with BD and MDD show deficits in cognitive control and processing biases toward negative materials ${ }^{11}$. Impaired cognitive control might lead to rumination tendency, which is a common feature of mood disorders, characterized by excessive focusing of attention on possible causes and consequences of one's distress ${ }^{12}$. Furthermore, rumination results in increased connectivity within the default-mode network (DMN) ${ }^{12,13}$, a set of brain areas related to self-referential processing and mind-wandering ${ }^{14,15}$. Under negative cognition, mind-wandering has been related to an increased tendency to ruminate in BD 
and MDD ${ }^{16}$. Therefore, the frequent occurrence of rumination during the resting-state could bring about altered recruitment of the DMN in patients with mood disorder. Interestingly, structural and functional abnormalities within the DMN brain areas have been documented in patients with BD 17-20 and MDD 21${ }^{24}$. However, a recent meta-analysis for MDD reported no abnormalities in the DMN ${ }^{15}$. Furthermore, a recent $f M R I$ study comparing $B D$ and MDD revealed that $D M N$ dysfunction was present to a greater extent in $\mathrm{BD}$ than in MDD ${ }^{25}$. Although further studies are needed to explore the DMN in BD and MDD, previous findings suggest that the $\mathrm{DMN}$ might play a significant role in the physiopathology of both $\mathrm{BD}$ and MDD.

To date, most of the previous studies have been performed using fMRI, which is a suitable imaging tool to investigate regional brain information owing to its excellent spatial resolution. However, fMRI lacks temporal resolution to elucidate neural processes occurring over the course of milliseconds ${ }^{26}$. Electroencephalography (EEG) is an appropriate tool to address the limitations of $\mathrm{fMRI}$ with high temporal resolution ${ }^{27}$. In addition, EEG is sensitive to alterations in neurotransmission secondary to pharmacological manipulations or brain dysfunction ${ }^{28}$. Resting-state brain activity reflects the baseline status of the brain and has been proposed for a means of exploring the underlying pathophysiology of mental disorders ${ }^{29}$. Unique resting-state EEG patterns have been observed in mental disorders, which is associated with the pathological characteristics of the disorders ${ }^{30,31}$. Thus, resting-state EEG analysis could help to better understand the pathophysiology of mental disorders.

Recently, an increasing number of researchers have studied cortical functional networks based on graph theory ${ }^{32,33}$. Graph theory-based brain network analysis could assist to comprehend the brain mechanisms of psychiatric disorders, such as mood disorders. However, to date, only a few studies have examined the resting-state cortical functional network in patients with BD or MDD using graph theory. For instance, Kim et al ${ }^{34}$ observed decreased CC and efficiency, and increased PL in the alpha band in patients with $\mathrm{BD}$. In addition, $\mathrm{BD}$ showed higher strength and $\mathrm{CC}$ and lower $\mathrm{PL}$ in the theta band ${ }^{35}$. In terms of MDD, lower PL and CC and greater efficiency have been reported ${ }^{36,37}$. In addition, decreased strength and CC and enhanced PL were observed in the alpha band in MDD ${ }^{38}$. Hasanzadeh et al ${ }^{39}$ indicated increased beta band global efficiency in MDD, while nodal level efficiency in MDD was almost the same as that in HCs. Furthermore, limited studies have directly compared the networks between BD and MDD. One of the studies comparing BD and MDD demonstrated that both disorders showed similar network changes with decreased CC and efficiency, and increased PL within the DMN and limbic system network ${ }^{40}$. Particularly, no study has investigated and compared their networks using EEG. The similarities and differences in brain functional networks between BD and MDD have been poorly understood. Therefore, further studies are needed to explore and compare their networks.

EEG has been known to lack spatial resolution coming from volume conduction ${ }^{41,42}$ and poor signal-tonoise ratio ${ }^{43,44}$. However, source imaging methods, advantageous alternatives to prevent the issue, can considerably enhance the spatial resolution of EEG. The objective of the current study was to investigate 
and compare cortical functional networks from resting-state EEG in patients with BD and MDD via source-level weighted network analysis. We also explored the associations between cortical network properties and psychological measures in patients with BD and MDD. We hypothesized that patients with $\mathrm{BD}$ and MDD would display altered cortical functional networks particularly in the DMN related regions, compared to healthy controls (HCs), and that two patient groups would show different degrees of alteration in their networks.

\section{Results}

\subsection{Demographic and psychological characteristics}

Table 1 presents the comparisons of demographic and psychological characteristics among the patients with BD and MDD and HCs. There was a significant difference in education years $(p<0.001)$. HCs showed significantly higher education years than patients with BD and MDD. In addition, there were significant differences in STAI state, STAI trait, and BDI (STAI state: $p<0.001$; STAI trait: $p<0.001$; BDI: $p$ $<0.001)$. HC presented significantly lower STAI state, STAI trait, and BDI scores than patients with BD and MDD. 
Table 1

Demographic characteristics of all study participants

\begin{tabular}{|c|c|c|c|c|c|}
\hline & $\begin{array}{l}\mathrm{BD} \\
(\mathrm{N}=35)\end{array}$ & $\begin{array}{l}\text { MDD } \\
(\mathrm{N}=39)\end{array}$ & $\begin{array}{l}\mathrm{HC} \\
(\mathrm{N}=42)\end{array}$ & $P$ & $\begin{array}{l}\text { Post-hoc } \\
\text { (Bonferroni) }\end{array}$ \\
\hline Age (years) & $\begin{array}{l}32.57 \pm \\
11.61\end{array}$ & $\begin{array}{l}31.62 \pm \\
12.28\end{array}$ & $\begin{array}{l}29.43 \pm \\
5.95\end{array}$ & 0.380 & \\
\hline Sex & & & & 0.412 & \\
\hline Male & $14(40.0)$ & $20(51.3)$ & $23(54.8)$ & & \\
\hline Female & $21(60.0)$ & $19(48.7)$ & $19(45.2)$ & & \\
\hline Education (years) & $12.97 \pm 2.53$ & $11.87 \pm 1.94$ & $\begin{array}{l}15.33 \pm \\
2.19\end{array}$ & $<001$ & $\begin{array}{l}\mathrm{BD}<\mathrm{HC}, \mathrm{MDD}< \\
\mathrm{HC}\end{array}$ \\
\hline Onset age (years) & $25.34 \pm 9.79$ & $\begin{array}{l}29.44 \pm \\
11.83\end{array}$ & & 0.112 & \\
\hline $\begin{array}{l}\text { Duration of illness } \\
\text { (years) }\end{array}$ & $7.26 \pm 7.71$ & $2.56 \pm 2.73$ & & 0.001 & \\
\hline STAI state & $60.69 \pm 9.54$ & $64.85 \pm 7.60$ & $\begin{array}{l}31.07 \pm \\
6.85\end{array}$ & $<.001$ & $\begin{array}{l}\mathrm{BD}>\mathrm{HC}, \mathrm{MDD}> \\
\mathrm{HC}\end{array}$ \\
\hline STAI trait & $62.06 \pm 9.91$ & $64.95 \pm 5.61$ & $\begin{array}{l}33.76 \pm \\
7.20\end{array}$ & $\dot{0} 001$ & $\begin{array}{l}\mathrm{BD}>\mathrm{HC}, \mathrm{MDD}> \\
\mathrm{HC}\end{array}$ \\
\hline BDI & $47.66 \pm 9.88$ & $\begin{array}{l}47.74 \pm \\
10.80\end{array}$ & $\begin{array}{l}23.38 \pm \\
3.93\end{array}$ & $<.001$ & $\begin{array}{l}\mathrm{BD}>\mathrm{HC}, \mathrm{MDD}> \\
\mathrm{HC}\end{array}$ \\
\hline
\end{tabular}

\subsection{Global level differences in cortical functional networks}

Table 2 presents the comparisons of global level indices among the BD, MDD, and HC groups. There were significant differences in the three global level indices of the high beta band. The strength $\left(p=0.001, \eta^{2}=\right.$ $0.112)$ and $C C\left(p=0.001, \eta^{2}=0.114\right)$ of the high beta band were significantly higher in patients with BD and MDD than in HCs. In contrast, the PL of the high beta band was significantly lower in patients with $\mathrm{BD}$ and MDD than in HCs $\left(p<0.001, \eta^{2}=0.129\right)$. There was no significant difference between the patient groups for the three network indices of the high beta band. Furthermore, there was no significant difference among the three groups in the other frequency bands. 
Table 2

Mean and standard deviation values of global network indices including strength, clustering coefficient (CC), and path length (PL) in each frequency band among the bipolar disorder, major depressive disorder, and healthy control groups

\begin{tabular}{|c|c|c|c|c|c|c|}
\hline & $\begin{array}{l}\mathrm{BD} \\
(\mathrm{N}=35)\end{array}$ & $\begin{array}{l}\text { MDD } \\
(N=39)\end{array}$ & $\begin{array}{l}\mathrm{HC} \\
(\mathrm{N}=42)\end{array}$ & $\begin{array}{l}\text { Effect size } \\
\left(n^{2}\right)\end{array}$ & $P^{*}$ & $\begin{array}{l}\text { Post-hoc } \\
\text { (Bonferroni) }\end{array}$ \\
\hline \multicolumn{7}{|c|}{ Delta band } \\
\hline Strength & $\begin{array}{l}28.02 \pm \\
1.26\end{array}$ & $\begin{array}{l}27.99 \pm \\
1.12\end{array}$ & $\begin{array}{l}27.67 \pm \\
0.89\end{array}$ & 0.006 & 0.702 & \\
\hline $\mathrm{CC}$ & $0.41 \pm 0.02$ & $0.41 \pm 0.02$ & $0.41 \pm 0.01$ & 0.006 & 0.728 & \\
\hline PL & $2.57 \pm 0.10$ & $2.57 \pm 0.09$ & $2.59 \pm 0.07$ & 0.004 & 0.802 & \\
\hline \multicolumn{7}{|c|}{ Theta band } \\
\hline Strength & $\begin{array}{l}24.51 \pm \\
1.40\end{array}$ & $\begin{array}{l}24.28 \pm \\
1.09\end{array}$ & $\begin{array}{l}23.91 \pm \\
0.87\end{array}$ & 0.023 & 0.274 & \\
\hline $\mathrm{CC}$ & $0.36 \pm 0.02$ & $0.35 \pm 0.02$ & $0.35 \pm 0.01$ & 0.022 & 0.282 & \\
\hline PL & $3.02 \pm 0.15$ & $3.04 \pm 0.12$ & $3.07 \pm 0.10$ & 0.019 & 0.344 & \\
\hline \multicolumn{7}{|c|}{ Alpha band } \\
\hline Strength & $\begin{array}{l}26.02 \pm \\
2.27\end{array}$ & $\begin{array}{l}26.97 \pm \\
2.99\end{array}$ & $\begin{array}{l}25.06 \pm \\
2.00\end{array}$ & 0.037 & 0.124 & \\
\hline $\mathrm{CC}$ & $0.38 \pm 0.03$ & $0.39 \pm 0.04$ & $0.36 \pm 0.03$ & 0.036 & 0.127 & \\
\hline PL & $2.84 \pm 0.24$ & $2.75 \pm 0.29$ & $2.94 \pm 0.21$ & 0.031 & 0.173 & \\
\hline \multicolumn{7}{|c|}{ Low beta band } \\
\hline Strength & $\begin{array}{l}21.22 \pm \\
1.08\end{array}$ & $\begin{array}{l}21.03 \pm \\
0.91\end{array}$ & $\begin{array}{l}20.40 \pm \\
0.93\end{array}$ & 0.077 & 0.012 & \\
\hline CC & $0.30 \pm 0.02$ & $0.30 \pm 0.01$ & $0.29 \pm 0.01$ & 0.077 & 0.011 & \\
\hline PL & $3.55 \pm 0.17$ & $3.59 \pm 0.14$ & $3.68 \pm 0.15$ & 0.080 & 0.009 & \\
\hline \multicolumn{7}{|l|}{$\begin{array}{l}\text { High beta } \\
\text { band }\end{array}$} \\
\hline Strength & $\begin{array}{l}17.54 \pm \\
1.14\end{array}$ & $\begin{array}{l}17.31 \pm \\
1.02\end{array}$ & $\begin{array}{l}16.54 \pm \\
0.85\end{array}$ & 0.112 & 0.001 & $\begin{array}{l}\mathrm{BD}>\mathrm{HC}, \\
\mathrm{MDD}>\mathrm{HC}\end{array}$ \\
\hline
\end{tabular}

* The $p$-value was adjusted via Bonferroni correction with $0.05 / 18=0.002778$.

BD, bipolar disorder; MDD, major depressive disorder; $\mathrm{HC}$, healthy control 


\begin{tabular}{|c|c|c|c|c|c|c|}
\hline & $\begin{array}{l}\mathrm{BD} \\
(\mathrm{N}=35)\end{array}$ & $\begin{array}{l}\text { MDD } \\
(N=39)\end{array}$ & $\begin{array}{l}\mathrm{HC} \\
(\mathrm{N}=42)\end{array}$ & $\begin{array}{l}\text { Effect size } \\
\left(n^{2}\right)\end{array}$ & $P^{*}$ & $\begin{array}{l}\text { Post-hoc } \\
\text { (Bonferroni) }\end{array}$ \\
\hline $\mathrm{CC}$ & $0.25 \pm 0.02$ & $0.24 \pm 0.01$ & $0.23 \pm 0.01$ & 0.114 & 0.001 & $\begin{array}{l}\mathrm{BD}>\mathrm{HC} \\
\mathrm{MDD}>\mathrm{HC}\end{array}$ \\
\hline PL & $4.38 \pm 0.30$ & $4.44 \pm 0.25$ & $4.67 \pm 0.24$ & 0.129 & $<0.001$ & $\begin{array}{l}\mathrm{BD}<\mathrm{HC} \\
\mathrm{MDD}<\mathrm{HC}\end{array}$ \\
\hline \multicolumn{7}{|c|}{ Gamma band } \\
\hline Strength & $\begin{array}{l}14.18 \pm \\
1.34\end{array}$ & $\begin{array}{l}14.29 \pm \\
1.28\end{array}$ & $\begin{array}{l}13.96 \pm \\
1.02\end{array}$ & 0.017 & 0.383 & \\
\hline $\mathrm{CC}$ & $0.19 \pm 0.02$ & $0.19 \pm 0.02$ & $0.19 \pm 0.01$ & 0.017 & 0.386 & \\
\hline PL & $5.29 \pm 0.55$ & $5.24 \pm 0.46$ & $5.38 \pm 0.43$ & 0.015 & 0.429 & \\
\hline \multicolumn{7}{|c|}{ * The $p$-value was adjusted via Bonferroni correction with $0.05 / 18=0.002778$. } \\
\hline BD, bipola & MDD, maj & epressive d & er; $\mathrm{HC}$, hea & ontrol & & \\
\hline
\end{tabular}

\subsection{Nodal level differences in cortical functional networks}

On the basis of the significant difference in the global high beta band CC among the three groups, we decided to explore possible differences at the nodal level in the high beta band. Figure 1 shows the violin plots of the significant nodal CCs in five regions among the three groups. The nodal CCs of the BD and MDD groups were significantly higher than that of HCs in one region (right precuneus, $p<0.001, \eta^{2}=$ 0.126). The nodal CCs of the BD were significantly higher than those of HCs in four regions (left isthmus cingulate: $p<0.001, \eta^{2}=0.144$; left paracentral: $p<0.001, \eta^{2}=0.131$; right paracentral: $p<0.001, \eta^{2}=$ 0.125 ; left superior frontal: $p<0.001, \eta^{2}=0.132$ ).

\subsection{Correlation between network indices and psychological characteristics}

The correlations between the network indices and psychological measures were evaluated in the high beta band. Only the patients with BD showed a significant correlations between them. At the global level, the strength was significantly positively correlated with the STAI state $(r=0.428, p=0.012)$ and STAI trait $(r=0.395, p=0.021)$. The CC was also significantly positively correlated with the STAl state $(r=0.425, p=$ $0.012)$ and STAI trait $(r=0.387, p=0.024)$. In contrast, the PL was significantly negatively correlated with the STAl state $(r=-0.403, p=0.018)$ and STAl trait $(r=-0.358, p=0.038)$. At the nodal level, the nodal CC in the right precuneus showed significant positive correlation with the STAI state $(r=0.356, p=0.039)$. In addition, the nodal $\mathrm{CC}$ in the left superior frontal region showed significant positive correlation with the STAl state $(r=0.392, p=0.022)$ and STAl trait $(r=0.367, p=0.033)$ (Fig. 2$)$. 


\section{Discussion}

This study investigated cortical functional networks during resting-state EEG in patients with BD and MDD and HCs. We observed significant differences among these groups in the high beta band. First, at the global level, strength and CC were significantly higher, while PL was lower in both patient groups compared with HCs. Second, at the nodal level, the CC in the right precuneus was significantly higher in both patient groups than that in HCs. Additionally, patients with BD showed also higher CCs in the left isthmus cingulate, bilateral paracentral, and left superior frontal, compared with HCs. Third, the nodal level high beta band CCs of the right precuneus and left superior frontal region correlated with the STAI state and/or trait in patients with BD.

Although only few studies have explored graph theory-based network properties in BD and MDD, they have been documented to have abnormal brain connectivity at the network-level. Structural brain networks from diffusion tensor imaging exhibited lower CC and efficiency, and longer PL in BD 45,46. Kim et al ${ }^{34}$ showed decreased CC and efficiency, and increased PL in the alpha band during resting-state EEG in patients with $\mathrm{BD}$. Another resting-state EEG study demonstrated that BD showed higher strength and $\mathrm{CC}$, and lower PL in the theta band ${ }^{35}$. Furthermore, several neuroimaging studies have shown altered functional brain networks in MDD patients. FMRI studies observed greater efficiency and lower PL and CC in MDD patients ${ }^{36,37,47}$. Guo et al ${ }^{48}$ reported that patients with MDD had stronger efficiency and shorter PL than HCs, but no difference was found at the local level between the MDD and HC groups. In addition, a resting-state EEG study ${ }^{39}$ indicated increased beta band global efficiency in patients with MDD, while nodal level efficiency in patients with MDD was almost the same as that in HCs. One resting-state fMRI study ${ }^{40}$ comparing BD and MDD patients demonstrated similar network changes in both patients with decreased CC and efficiency, and increased PL compared to HCs within the DMN and limbic system network. Although the previous results tend to be inconsistent regarding the direction of altered network properties in the disorders, these findings support our results that patients with BD and MDD displayed similar abnormal cortical functional networks at the global level.

Although previous studies did not show a consensus of abnormality in a specific frequency band in patients with $B D$ and MDD, various studies have reported that the beta band is closely related to the patients. Several EEG studies have concluded that beta power positively correlates with the DMN activity in $\mathrm{BD}^{49-51}$. A recent magnetoencephalographic (MEG) study ${ }^{52}$ revealed that the limbic network connectivity in the beta band could be a good objective biomarker for BD. In terms of MDD, one EEG study 53 revealed that patients with MDD showed greater beta band functional connectivity within the DMN. In addition, MEG studies have reported the association of altered connectivity in the beta band with MDD 54,55 . Excessive beta band synchronization, which is associated with maintaining the brain's status quo, might be a mechanism driving brain inflexibility in BD and MDD patients. Previous studies back up our findings that excessive neural processing in the beta band might be associated with the pathophysiology in depressive states in mood disorders. 
At the nodal level, our results showed that compared to HCs, patients with BD showed higher nodal CCs of the high beta band in the right precuneus, left isthmus cingulate, bilateral paracentral, and left superior frontal, which belonged to the DMN. In contrast, compared to HCs, patients with MDD showed higher nodal CC only in the right precuneus. These findings might imply that patients with BD could be associated with a more severely altered network in DMN-related regions. Our results are in line with some previous studies where MDD patients showed altered global network properties, while they presented almost the same nodal level network as that of $\mathrm{HCs}^{39,48}$. However, these studies only included patients with MDD. To verify our findings, further studies with both patient groups are necessary.

DMN is known to include several regions, such as the posterior cingulate, isthmus cingulate, precuneus, paracentral gyrus, inferior frontal lobe, superior frontal gyrus, and inferior parietal lobe ${ }^{17,56,57}$. Aberrant DMN function could lead to dysfunctional self-referential and affective processing with an excessively negative self-focus ${ }^{58}$. Previous studies have repeatedly shown an abnormality in the precuneus, the core region of the $D M N$, in both $B D$ and MDD patients. BD patients showed a lack of precuneus activation during emotion related paradigms ${ }^{59,60}$. A resting-state fMRI study ${ }^{61}$ found increased neuronal synchronization in the right precuneus, left superior frontal gyrus, and right paracentral lobule, indicating that these areas might play an important role in the pathophysiology of $\mathrm{BD}$. In terms of MDD, lower activity in the right precuneus was observed relative to the HCs ${ }^{62,63}$. Zhu et al ${ }^{23}$ found decreased connectivity of the precuneus to the DMN in patients with MDD compared to HCs. In addition, a recent fMRI study found that reduced strength of the right precuneus was associated with higher maladaptive rumination levels in patients with MDD ${ }^{64}$. Among the few studies directly comparing both BD and MDD patients, Liang et al ${ }^{65}$ found that the regional homogeneity value of the precuneus was increased in $\mathrm{BD}$ and MDD patients. Another fMRI study ${ }^{40}$ found similar network changes between BD and MDD, but abnormal nodal efficiency in the right precuneus was specific to BD patients.

Moreover, evidence of abnormalities in other DMN-related regions besides the precuneus has been accumulated in BD. For example, a diffusion-weighted MRI study ${ }^{45}$ revealed abnormalities of nodal network measures in the bilateral isthmus cingulate in BD. Another structural brain network study 56 identified alterations in community structure containing the paracentral gyrus and posterior cingulate in $B D$. They also found that the left isthmus cingulate alteration was associated with the number of depressive episodes in patients with BD. With regard to the superior frontal region, cortical thinning or volume reduction in the left superior frontal cortex, a key emotional processing region, has been observed in $\mathrm{BD}$ patients ${ }^{66,67}$. A diffusion tensor imaging study reported reduced fractional anisotropy in the superior frontal white matter in BD patients ${ }^{68}$. Regarding the paracentral region, patients with BD showed increased gray matter volume in the bilateral paracentral lobule ${ }^{69}$. An fMRI study ${ }^{70}$ reported reduced nodal degree and increased participation coefficient in the paracentral lobule in BD. These studies support our findings that the regions belonging to the DMN could play a crucial role in comprehending the pathophysiology of both patient groups, particularly in BD patients. Furthermore, our findings might imply 
that the network of BD patients could be more functionally altered in the DMN than that in patients with MDD.

Interestingly, our findings revealed intermediate network measures of patients with MDD, between those in patients with $\mathrm{BD}$ and those in $\mathrm{HCs}$, although the two patient groups did not show significantly different network measures. Our BD patient population was composed of more BD Type II patients than BD Type I patients (25 patients with BD Type II and 10 patients with BD Type I). BD II is distinguished from BD I mainly by the absence of full-blown manic episodes, and a growing body of evidence suggests that there could be neurobiological differences between BD I and BD II patients ${ }^{71}$. In addition, according to a previous study, the DMN might reflect overlapping pathophysiological processes in patients with BD II and MDD ${ }^{40}$. Therefore, the higher proportion of patients with BD II in the present study might have affected our results that there were no significant differences in network measures between the two patient groups. Furthermore, the difference between BD and MDD patients might not be detected because the current study population did not include patients with psychotic symptoms. The comorbid psychotic features are usually related to low functioning and disability ${ }^{72}$ and worse cognitive performance in BD 73 . Further research needs to be conducted with a large sample size of both BD subtypes and various symptom dimensions, such as psychotic features.

Moreover, our significant correlation results were found only in BD patients where the nodal level high beta band CCs of the right precuneus and left superior frontal region were positively correlated with state and trait anxiety. One possible explanation for the results could be the more altered network in BD than MDD. In other words, although both patient groups did not show differences in phenotypic anxiety levels through self-reports, more altered network in the DMN-related regions in the patients with BD could have been more affected by state and trait anxiety. Increased connectivity in the DMN is associated with ruminative thoughts and excessive worries during self-referential processing ${ }^{74}$. Thus, the link between nodal CCs in DMN-related regions and state and trait anxiety suggests more negative internalized attention in patients with $\mathrm{BD}^{15}$. Furthermore, the high beta band frequency domain has been shown to be related to anxiety ${ }^{75,76}$. The altered DMN activity in the beta frequency band was repeatedly observed in patients with BD ${ }^{49-51}$. Taken together, the disrupted beta band network in DMN-related regions in BD patients might contribute to affective functioning underlying clinical symptoms such as anxiety.

This study has a few limitations. First, some of the patients were taking medications at the time of testing. However, less than $30 \%$ of the patients were taking medications in each group (9 patients with BD and 11 patients with MDD). Further studies controlling for the medication effects would be needed. Second, the current study enrolled patients with depression but without psychotic symptoms. Thus, our findings may not be generalized to the entire population of individuals with BD and MDD. Third, we did not use individual head models for EEG source imaging. Source analysis of scalp-derived EEG might be inherently limited because of the precision of spatial localization. Furthermore, we not only focused on the regions of the DMN, but we explored whole brain networks. To observe the exact DMN connectivity or network measures, further study directly focusing on the DMN is necessary. Despite these limitations, this 
study was the first attempt to investigate and compare source-level cortical functional networks in patients with BD and MDD during resting-state EEG. Our results suggest that both patients have similar network changes at the global level, but they have different network changes related to the DMN regions at the nodal level. We also found significant correlations between cortical network states and anxietyrelated psychological measures in BD patients. Our source-level cortical network indices might contribute to the understanding of the neuropathological mechanisms in these two disorders. Further studies with larger sample sizes are necessary to replicate and extend the generalizability of our findings.

\section{Materials And Methods}

\subsection{Participants}

Participants were recruited from the Department of Psychiatry at Soonchunhyang University Cheonan Hospital in Korea. The patients included 35 patients with BD (14 men and 21 women; mean age: $32.57 \pm$ 11.61 years) and 39 patients with MDD (20 men and 19 women; mean age: $31.54 \pm 12.32$ years). Patients with BD and MDD were diagnosed according to the Structured Clinical Interview for Diagnostic and Statistical Manual of Mental Disorders, 4th edition (DSM-IV) Axis I Psychiatric Disorders by a boardcertified psychiatrist ${ }^{77}$. Patients with any history of neurological or other severe medical diseases were excluded in the initial screening interviews. None of the patients had mental retardation, alcohol abuse, were undergoing electroconvulsive therapy, or had any head injuries. Among the 35 patients with BD, 10 patients were diagnosed with BD Type I and 25 patients with BD Type II. Nine of the 35 patients with BD were taking mood-stabilizing agents (lithium and valproate) with or without atypical antipsychotics (quetiapine, aripiprazole, and olanzapine). Eleven of the 39 patients with MDD were taking medications, such as selective serotonin reuptake inhibitors (fluoxetine and escitalopram), serotonin, norepinephrine reuptake inhibitors (duloxetine), or others (mirtazapine). Forty-two HCs (23 men and 19 women; mean age: $29.43 \pm 5.95$ years) were recruited through posters in the hospital and advertisements in local newspapers. An initial screening interview was conducted by a board-certified psychiatrist to exclude any subjects with identifiable psychiatric disorders, neurological disorders, or histories of head injuries. All participants were right-handed. This study was approved by the Institutional Review Board and Ethics Committee of Soonchunhyang University Cheonan Hospital, and all experimental protocols were approved by the committee (2018-10-032-006). The study was performed in accordance with approved guidelines. Informed consent was acquired from all study participants.

\subsection{Psychological measures}

The State-Trait Anxiety Inventory (STAI) ${ }^{78,79}$ and Beck Depression Inventory (BDI) ${ }^{80}$ were evaluated for anxiety and depression. The STAl is a self-rating scale for state and trait anxiety ${ }^{79}$. It consists of a state anxiety inventory and trait anxiety inventory; each inventory has 20 items ${ }^{78}$. The BDI is a self-rating scale composed of 21 items to measure the severity of depression symptoms ${ }^{80}$.

\subsection{Recording and preprocessing of electroencephalography (EEG)}


Resting-state EEG data were recorded in a sound-attenuated room, while the participants opened their eyes for five minutes. EEG was recorded with a NeuroScan SynAmps2 amplifier (Compumedics USA, Charlotte, NC, USA) based on an extended 10-20 placement scheme via $62 \mathrm{Ag}-\mathrm{AgCl}$ electrodes mounted on a Quik-Cap. The reference electrode was $\mathrm{Cz}$ and the ground electrode was located on the forehead. Horizontal electrooculogram (EOG) electrodes were placed at the outer canthus of each eye while vertical EOG electrodes were located above and below the left eye. The impedance was kept below $5 \mathrm{k} \Omega$. EEG signals were bandpass filtered from 0.1 to $100 \mathrm{~Hz}$ with a $1,000-\mathrm{Hz}$ sampling rate. The procedure for the EEG recording followed our previous study ${ }^{35}$.

All preprocessing procedures were performed using CURRY 8 (Compumedics USA, Charlotte, NC, USA) and MATLAB R2018b (MathWorks, Natick, MA, USA). The EEG data were re-referenced to an average reference. In order to remove DC components, a high pass filter with $1 \mathrm{~Hz}$ was applied to the EEG data. Visual inspection for movement artifacts was carried out by a skilled researcher without prior information regarding the data origin. Eye-movement related artifacts were corrected via a covariance- and regressionbased mathematical procedure in CURRY $8{ }^{81}$. Then, the preprocessed EEG data were divided into $2 \mathrm{~s}$ (2,048 points) epochs, and all the epochs including significant physiological artifacts (amplitude exceeding $\pm 100 \mu \mathrm{V}$ ) at any of the 62 electrodes were rejected. Finally, each participant had 30 artifactfree epochs. It was determined by the different number of remaining epochs from each participant after rejecting artifacts. In addition, a previous study demonstrated acceptable reliability of resting-state EEG data longer than 40 seconds 82 .

\subsection{Source localization}

The depth-weighted minimum L2 norm estimator from the Brainstorm toolbox (http://neuroimage.usc.edu/brainstorm) was used to approximate the time series of source activities ${ }^{83}$. A three-layer boundary element model from the MNI/Colin 27 anatomy template was used to compute the lead-field matrix. Cortical current density of 15,002 cortical vertices was calculated at every time point of each epoch. Noise covariance was measured by the whole 30 epochs of each participant. The weight of each individual sensor was computed by the diagonal components of the noise covariance for the source reconstruction. After approximating the cortical current density at every time point, 68 nodes were extracted based on the Desikan-Killiany atlas containing 34 cortical regions in each hemisphere ${ }^{84}$. The representative value in each region was assessed by the cortical source of the seed point in each region based on the Desikan-Killiany atlas. Then, the time series of the cortical sources at each of the 68 seed points were bandpass filtered and divided into six frequency bands: delta $(1-4 \mathrm{~Hz})$, theta $(4-8 \mathrm{~Hz})$, alpha (8-12 Hz), low beta $(12-18 \mathrm{~Hz})$, high beta $(18-30 \mathrm{~Hz})$, and gamma $(30-55 \mathrm{~Hz})$. The procedure for the source localization followed that of our previous study ${ }^{35}$.

\subsection{Connectivity and network analysis}

Functional connectivity between each pair of nodes was assessed via phase-locking values (PLVs) ${ }^{85}$. PLVs provide normalized synchronization values ranging from 0 to 1 . Thus, no further modification was needed to apply them to the weighted network analysis. A higher PLV denotes stronger connection 
between two nodes than that between the other pairs. PLV showed fine performance with weighted minimum norm estimation ${ }^{86}$ and has been widely employed in network analysis ${ }^{87,88}$.

We performed a graph theory-based weighted network analysis ${ }^{32,33}$. The weighted network preserves the unique traits of the original network without distortion. A network is comprised of several nodes connecting to each other using edges. Widely used three representative global level weighted network measures were evaluated in this study. First, "strength" denotes the degree of connection strength in the network. A higher strength value indicates the strong connection in the whole brain. Second, "CC" refers to the degree to which a node clusters with its neighboring nodes. An increased CC denotes a wellsegregated network between the relevant brain regions. Third, "PL" denotes the sum of lengths between two nodes within the network. It is associated with the information processing speed. The shortened $\mathrm{PL}$ refers to a well-integrated network. Furthermore, weighted nodal CC was assessed at each node. Network analyses were carried out via MATLAB R2018b.

\subsection{Statistical analysis}

Chi-squared tests and one-way analysis of variance (ANOVA) were used to explore differences in demographic characteristics and psychological measures among the three groups. A multivariate ANOVA was conducted to compare the cortical network characteristics at the global level of each frequency band among the three groups, with education as a covariate. $P$-values were adjusted by Bonferroni corrections (an adjusted $p$-value of $0.05 / 18=0.002778$; three global network measures with six frequency bands) to control multiple comparisons. The same analysis was conducted at the nodal level, followed by Bonferroni corrections with an adjusted $p$-value of $0.05 / 68=0.000735$ (nodal CCs at 68 nodes). When the variables presented significant differences among the three groups, the post-hoc pair-wise comparisons with Bonferroni corrections were performed. Effect sizes were computed based on the partial eta squared $\left(\eta^{2}\right)$. The procedure for the statistical analysis followed our previous study ${ }^{35}$.

A partial Pearson's correlation was carried out between network indices and psychological measures, with a 5,000-bootstrap resampling technique to correct multiple correlations in each group. For the patient groups, the duration of illness was considered as a covariate. Although the bootstrap test is weaker than the Bonferroni test to resolve the multiple-comparison issue, the robustness and stability of the bootstrap test have been verified ${ }^{89,90}$. Furthermore, the bootstrap test has been widely used in EEG analysis ${ }^{91,92}$. Statistical analyses were performed using SPSS 21 with the significant level at $p<0.05$ (two-tailed) (SPSS, Inc., Chicago, IL, USA).

\section{Declarations}

\section{Acknowledgements}

This work was supported by a grant (2020R1/1A3068017) awarded by the Basic Science Research Program through the National Research Foundation of Korea (NRF) and funded by the Ministry of Education. This study was also supported by Soonchunhyang University. 


\section{Author Contributions}

S.K. analysed the data and wrote the paper. J.S.K. designed the study and wrote the paper. S.H.S, Y.J.K, and H.Y.L collected the data. J.S.K. and J.H.B and J.H.Y reviewed and revised the paper.

\section{Conflict of Interest}

All authors in this study declare that they have no conflicts of interest.

\section{References}

1 Fung, G. et al. Distinguishing bipolar and major depressive disorders by brain structural morphometry: a pilot study. BMC psychiatry 15, 298 (2015).

2 Patella, A. M. et al. Clinical features of differential diagnosis between unipolar and bipolar depression in a drug-free sample of young adults. Journal of affective disorders 243, 103-107 (2019).

3 Kempton, M. J. et al. Structural neuroimaging studies in major depressive disorder: meta-analysis and comparison with bipolar disorder. Archives of general psychiatry 68, 675-690 (2011).

4 Delvecchio, G. et al. Common and distinct neural correlates of emotional processing in bipolar disorder and major depressive disorder: a voxel-based meta-analysis of functional magnetic resonance imaging studies. European Neuropsychopharmacology 22, 100-113 (2012).

5 Vargas, C., López-Jaramillo, C. \& Vieta, E. A systematic literature review of resting state networkfunctional MRI in bipolar disorder. Journal of affective disorders 150, 727-735 (2013).

6 Gong, Q. \& He, Y. Depression, neuroimaging and connectomics: a selective overview. Biological psychiatry 77, 223-235 (2015).

7 de Almeida, J. R. C. \& Phillips, M. L. Distinguishing between unipolar depression and bipolar depression: current and future clinical and neuroimaging perspectives. Biological psychiatry 73, 111-118 (2013).

8 Marchand, W. R., Lee, J. N., Johnson, S., Gale, P. \& Thatcher, J. Differences in functional connectivity in major depression versus bipolar II depression. Journal of affective disorders 150, 527-532 (2013).

9 Anand, A., Li, Y., Wang, Y., Lowe, M. J. \& Dzemidzic, M. Resting state corticolimbic connectivity abnormalities in unmedicated bipolar disorder and unipolar depression. Psychiatry Research: Neuroimaging 171, 189-198 (2009).

10 Wang, Y. et al. Interhemispheric resting state functional connectivity abnormalities in unipolar depression and bipolar depression. Bipolar disorders 17, 486-495 (2015). 
11 Joormann, J. \& Gotlib, I. H. Emotion regulation in depression: Relation to cognitive inhibition. Cognition and Emotion 24, 281-298 (2010).

12 Apazoglou, K. et al. Rumination related activity in brain networks mediating attentional switching in euthymic bipolar patients. International journal of bipolar disorders 7, 3 (2019).

13 Cooney, R. E., Joormann, J., Eugène, F., Dennis, E. L. \& Gotlib, I. H. Neural correlates of rumination in depression. Cognitive, Affective, \& Behavioral Neuroscience 10, 470-478 (2010).

14 Mason, M. F. et al. Wandering minds: the default network and stimulus-independent thought. Science 315, 393-395 (2007).

15 Hamilton, J. P., Farmer, M., Fogelman, P. \& Gotlib, I. H. Depressive rumination, the default-mode network, and the dark matter of clinical neuroscience. Biological psychiatry 78, 224-230 (2015).

16 Marchetti, I., Koster, E. H., Klinger, E. \& Alloy, L. B. Spontaneous thought and vulnerability to mood disorders: The dark side of the wandering mind. Clinical Psychological Science 4, 835-857 (2016).

17 Buckner, R. L., Andrews-Hanna, J. R. \& Schacter, D. L. The brain's default network: anatomy, function, and relevance to disease. (2008).

18 Liu, C.-H. et al. Regional homogeneity within the default mode network in bipolar depression: a resting-state functional magnetic resonance imaging study. PloS one 7, e48181 (2012).

19 Alonso-Lana, S. et al. Longitudinal brain functional changes between mania and euthymia in bipolar disorder. Bipolar disorders 21, 449-457 (2019).

20 Gong, J. et al. Disrupted functional connectivity within the default mode network and salience network in unmedicated bipolar II disorder. Progress in Neuro-Psychopharmacology and Biological Psychiatry 88, 11-18 (2019).

21 Sheline, Y. I. et al. The default mode network and self-referential processes in depression. Proceedings of the National Academy of Sciences 106, 1942-1947 (2009).

22 Hamilton, J. P. et al. Default-mode and task-positive network activity in major depressive disorder: implications for adaptive and maladaptive rumination. Biological psychiatry 70, 327-333 (2011).

23 Zhu, X. et al. Evidence of a dissociation pattern in resting-state default mode network connectivity in first-episode, treatment-naive major depression patients. Biological psychiatry 71, 611-617 (2012).

24 Korgaonkar, M. S., Fornito, A., Williams, L. M. \& Grieve, S. M. Abnormal structural networks characterize major depressive disorder: a connectome analysis. Biological psychiatry 76, 567-574 (2014).

25 Rodríguez-Cano, E. et al. Differential failure to deactivate the default mode network in unipolar and bipolar depression. Bipolar disorders 19, 386-395 (2017). 
26 Kim, S. G., Richter, W. \& Uğurbil, K. Limitations of temporal resolution in functional MRI. Magnetic resonance in medicine 37, 631-636 (1997).

27 Burle, B. et al. Spatial and temporal resolutions of EEG: Is it really black and white? A scalp current density view. International Journal of Psychophysiology 97, 210-220 (2015).

28 Luck, S. J. et al. A roadmap for the development and validation of event-related potential biomarkers in schizophrenia research. Biological psychiatry 70, 28-34 (2011).

29 Liu, C.-H. et al. Abnormal baseline brain activity in bipolar depression: a resting state functional magnetic resonance imaging study. Psychiatry Research: Neuroimaging 203, 175-179 (2012).

30 Hughes, J. R. \& John, E. R. Conventional and quantitative electroencephalography in psychiatry. The Journal of Neuropsychiatry and Clinical Neurosciences 11, 190-208 (1999).

31 Sponheim, S. R., Clementz, B. A., lacono, W. G. \& Beiser, M. Clinical and biological concomitants of resting state EEG power abnormalities in schizophrenia. Biological psychiatry 48, 1088-1097 (2000).

32 Bullmore, E. \& Sporns, O. Complex brain networks: graph theoretical analysis of structural and functional systems. Nature reviews. Neuroscience 10, 186 (2009).

33 Rubinov, M. \& Sporns, O. Complex network measures of brain connectivity: uses and interpretations. Neuroimage 52, 1059-1069 (2010).

$34 \mathrm{Kim}, \mathrm{D}$.-J. et al. Disturbed resting state EEG synchronization in bipolar disorder: a graph-theoretic analysis. Neurolmage: Clinical 2, 414-423 (2013).

35 Kim, S. et al. Altered Cortical Functional Networks in Patients With Schizophrenia and Bipolar Disorder: A Resting-State Electroencephalographic Study. Frontiers in psychiatry 11, 661 (2020).

36 Bohr, I. J. et al. Resting-state functional connectivity in late-life depression: higher global connectivity and more long distance connections. Frontiers in psychiatry 3, 116 (2013).

37 Li, H., Zhou, H., Yang, Y., Wang, H. \& Zhong, N. More randomized and resilient in the topological properties of functional brain networks in patients with major depressive disorder. Journal of Clinical Neuroscience 44, 274-278 (2017).

38 Shim, M., Im, C.-H., Kim, Y.-W. \& Lee, S.-H. Altered cortical functional network in major depressive disorder: A resting-state electroencephalogram study. Neurolmage: Clinical 19, 1000-1007 (2018).

39 Hasanzadeh, F., Mohebbi, M. \& Rostami, R. Graph theory analysis of directed functional brain networks in major depressive disorder based on EEG signal. Journal of Neural Engineering 17, 026010 (2020). 
40 Wang, Y. et al. Topologically convergent and divergent functional connectivity patterns in unmedicated unipolar depression and bipolar disorder. Translational Psychiatry 7, e1165-e1165 (2017).

41 van den Broek, S. P., Reinders, F., Donderwinkel, M. \& Peters, M. Volume conduction effects in EEG and MEG. Electroencephalography and clinical neurophysiology 106, 522-534 (1998).

42 Nolte, G. et al. Identifying true brain interaction from EEG data using the imaginary part of coherency. Clinical neurophysiology 115, 2292-2307 (2004).

43 Lange, D. H. \& Inbar, G. F. A robust parametric estimator for single-trial movement related brain potentials. IEEE transactions on biomedical engineering 43, 341-347 (1996).

44 Lemm, S., Curio, G., Hlushchuk, Y. \& Muller, K.-R. Enhancing the signal-to-noise ratio of ICA-based extracted ERPs. IEEE Transactions on Biomedical Engineering 53, 601-607 (2006).

45 Leow, A. et al. Impaired inter-hemispheric integration in bipolar disorder revealed with brain network analyses. Biological psychiatry 73, 183-193 (2013).

46 Collin, G. et al. Brain network analysis reveals affected connectome structure in bipolar I disorder. Human brain mapping 37, 122-134 (2016).

47 Zhang, J. et al. Disrupted brain connectivity networks in drug-naive, first-episode major depressive disorder. Biological psychiatry 70, 334-342 (2011).

48 Guo, H. et al. Resting-state functional connectivity abnormalities in first-onset unmedicated depression. Neural regeneration research 9, 153 (2014).

49 Laufs, H. et al. Electroencephalographic signatures of attentional and cognitive default modes in spontaneous brain activity fluctuations at rest. Proceedings of the national academy of sciences 100 , 11053-11058 (2003).

50 Mantini, D., Perrucci, M. G., Del Gratta, C., Romani, G. L. \& Corbetta, M. Electrophysiological signatures of resting state networks in the human brain. Proceedings of the National Academy of Sciences 104, 13170-13175 (2007).

51 Hlinka, J., Alexakis, C., Diukova, A., Liddle, P. F. \& Auer, D. P. Slow EEG pattern predicts reduced intrinsic functional connectivity in the default mode network: an inter-subject analysis. Neuroimage 53, 239-246 (2010).

52 Sunaga, M. et al. Frequency-Specific Resting Connectome in Bipolar Disorder: An MEG Study. Frontiers in psychiatry 11, 597 (2020).

53 Whitton, A. E. et al. Electroencephalography source functional connectivity reveals abnormal highfrequency communication among large-scale functional networks in depression. Biological Psychiatry: 
Cognitive Neuroscience and Neuroimaging 3, 50-58 (2018).

54 Salvadore, G. et al. Anterior cingulate desynchronization and functional connectivity with the amygdala during a working memory task predict rapid antidepressant response to ketamine. Neuropsychopharmacology 35, 1415-1422 (2010).

55 Nugent, A. C., Robinson, S. E., Coppola, R., Furey, M. L. \& Zarate Jr, C. A. Group differences in MEGICA derived resting state networks: Application to major depressive disorder. Neuroimage 118, 1-12 (2015).

56 GadElkarim, J. J. et al. Investigating brain community structure abnormalities in bipolar disorder using path length associated community estimation. Human brain mapping 35, 2253-2264 (2014).

57 Park, H.-J., Friston, K. J., Pae, C., Park, B. \& Razi, A. Dynamic effective connectivity in resting state fMRI. Neuroimage 180, 594-608 (2018).

58 Whitfield-Gabrieli, S. \& Ford, J. M. Default mode network activity and connectivity in psychopathology. Annual review of clinical psychology 8, 49-76 (2012).

59 Malhi, G. S., Lagopoulos, J., Sachdev, P. S., Ivanovski, B. \& Shnier, R. An emotional Stroop functional MRI study of euthymic bipolar disorder. Bipolar disorders 7, 58-69 (2005).

60 Tesli, M. et al. Altered brain activation during emotional face processing in relation to both diagnosis and polygenic risk of bipolar disorder. PloS one 10, e0134202 (2015).

61 Achalia, R. M. et al. Investigating spontaneous brain activity in bipolar disorder: A resting-state functional magnetic resonance imaging study. Indian Journal of Psychiatry 61, 630 (2019).

62 Jing, B. et al. Difference in amplitude of low-frequency fluctuation between currently depressed and remitted females with major depressive disorder. Brain research 1540, 74-83 (2013).

$63 \mathrm{Li}, \mathrm{G} .$, Rossbach, K., Zhang, A., Liu, P. \& Zhang, K. Resting-state functional changes in the precuneus within first-episode drug-naive patients with MDD. Neuropsychiatric disease and treatment 14, 1991 (2018).

64 Jacob, Y. et al. Neural correlates of rumination in major depressive disorder: A brain network analysis. Neurolmage: Clinical 25, 102142 (2020).

65 Liang, M.-J. et al. Identify changes of brain regional homogeneity in bipolar disorder and unipolar depression using resting-state FMRI. PloS one 8, e79999 (2013).

66 Hanford, L. C., Nazarov, A., Hall, G. B. \& Sassi, R. B. Cortical thickness in bipolar disorder: a systematic review. Bipolar disorders 18, 4-18 (2016). 
67 Wang, X. et al. Brain grey-matter volume alteration in adult patients with bipolar disorder under different conditions: a voxel-based meta-analysis. Journal of psychiatry \& neuroscience: JPN 44, 89 (2019).

68 Benedetti, F. et al. Disruption of white matter integrity in bipolar depression as a possible structural marker of illness. Biological psychiatry 69, 309-317 (2011).

69 Adler, C. M. et al. Voxel-based study of structural changes in first-episode patients with bipolar disorder. Biological psychiatry 61, 776-781 (2007).

70 Doucet, G. E., Bassett, D. S., Yao, N., Glahn, D. C. \& Frangou, S. The role of intrinsic brain functional connectivity in vulnerability and resilience to bipolar disorder. American Journal of Psychiatry 174, 12141222 (2017).

71 Abé, C. et al. Cortical thickness, volume and surface area in patients with bipolar disorder types I and II. Journal of psychiatry \& neuroscience: JPN 41, 240 (2016).

72 Goldberg, J. F. \& Harrow, M. Poor-outcome bipolar disorders. Bipolar disorders: Clinical course and outcome, 1-19 (1999).

73 Glahn, D. C. et al. The neurocognitive signature of psychotic bipolar disorder. Biological psychiatry 62, 910-916 (2007).

74 Lemogne, C. et al. In search of the depressive self: extended medial prefrontal network during selfreferential processing in major depression. Social cognitive and affective neuroscience 4, 305-312 (2009).

75 Ribas, V. R. et al. Pattern of anxiety, insecurity, fear, panic and/or phobia observed by quantitative electroencephalography (QEEG). Dementia \& neuropsychologia 12, 264-271 (2018).

76 Díaz, H. et al. EEG Beta band frequency domain evaluation for assessing stress and anxiety in resting, eyes closed, basal conditions. Procedia Computer Science 162, 974-981 (2019).

77 First, M. B., Gibbon, M., Spitzer, R. L. \& Benjamin, L. S. User's guide for the structured clinical interview for DSM-IV axis II personality disorders: SCID-II. (American Psychiatric Pub, 1997).

$78 \mathrm{Kim}, \mathrm{J}$. \& Shin, D. A study based on the standardization of the STAI for Korea. New Med J 21, 6975 (1978).

79 Spielberger, C. D. Manual for the State-Trait Anxiety Inventory STAI (form Y)(" self-evaluation questionnaire"). (1983).

80 Lee, M. et al. A standardization study of beck depression inventory (I): Korean version (K-BDI): reliability land factor analysis. Korean J Psychopathol 4, 77-95 (1995). 
81 Semlitsch, H. V., Anderer, P., Schuster, P. \& Presslich, O. A solution for reliable and valid reduction of ocular artifacts, applied to the P300 ERP. Psychophysiology 23, 695-703 (1986).

82 Gudmundsson, S., Runarsson, T. P., Sigurdsson, S., Eiriksdottir, G. \& Johnsen, K. Reliability of quantitative EEG features. Clinical Neurophysiology 118, 2162-2171 (2007).

83 Tadel, F., Baillet, S., Mosher, J. C., Pantazis, D. \& Leahy, R. M. Brainstorm: a user-friendly application for MEG/EEG analysis. Computational intelligence and neuroscience 2011, 8 (2011).

84 Desikan, R. S. et al. An automated labeling system for subdividing the human cerebral cortex on MRI scans into gyral based regions of interest. Neuroimage 31, 968-980 (2006).

85 Lachaux, J.-P., Rodriguez, E., Martinerie, J. \& Varela, F. J. Measuring phase synchrony in brain signals. Human brain mapping 8, 194-208 (1999).

86 Hassan, M., Dufor, O., Merlet, I., Berrou, C. \& Wendling, F. EEG source connectivity analysis: from dense array recordings to brain networks. PloS one $\mathbf{9}$ (2014).

87 Gong, A. et al. Characteristic differences between the brain networks of high-level shooting athletes and non-athletes calculated using the phase-locking value algorithm. Biomedical Signal Processing and Contro/ 51, 128-137 (2019).

$88 \mathrm{Li}$, P. et al. EEG based emotion recognition by combining functional connectivity network and local activations. IEEE Transactions on Biomedical Engineering 66, 2869-2881 (2019).

89 Ruscio, J. Constructing confidence intervals for Spearman's rank correlation with ordinal data: a simulation study comparing analytic and bootstrap methods. Journal of Modern Applied Statistical Methods 7, 7 (2008).

90 Pernet, C. R., Wilcox, R. R. \& Rousselet, G. A. Robust correlation analyses: false positive and power validation using a new open source Matlab toolbox. Frontiers in psychology 3, 606 (2013).

91 Pernet, C. R., Chauveau, N., Gaspar, C. \& Rousselet, G. A. LIMO EEG: a toolbox for hierarchical LInear MOdeling of ElectroEncephaloGraphic data. Computational intelligence and neuroscience 2011, 3 (2011).

92 Kim, J. S., Kim, S., Jung, W., Im, C.-H. \& Lee, S.-H. Auditory evoked potential could reflect emotional sensitivity and impulsivity. Scientific reports 6, 37683 (2016).

\section{Figures}


High beta band nodal-level CCs

- Left lsthmus cingulate $(p<0.001)$

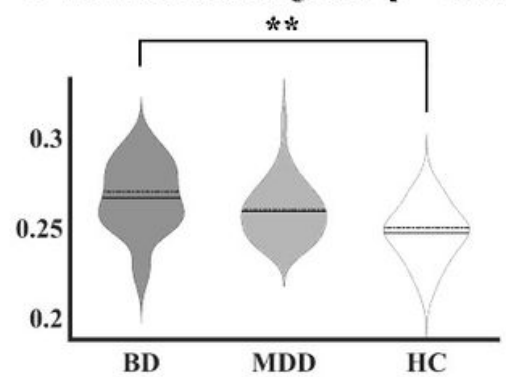

Left Paracentral $(p<0.001)$

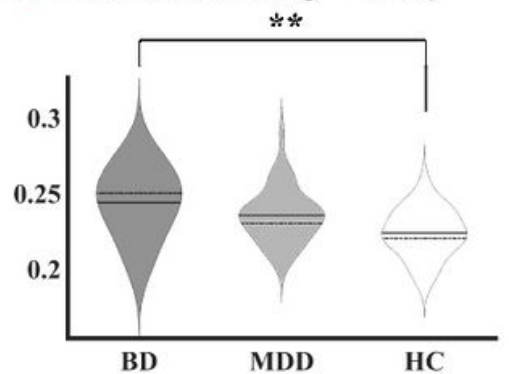

Right Paracentral $(p<0.001)$

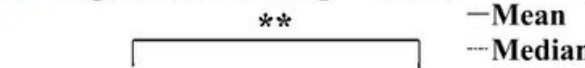

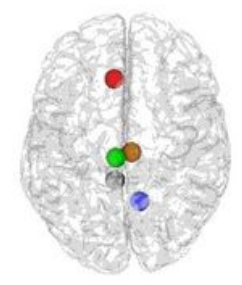

Right Precuneus $(p<0.001)$

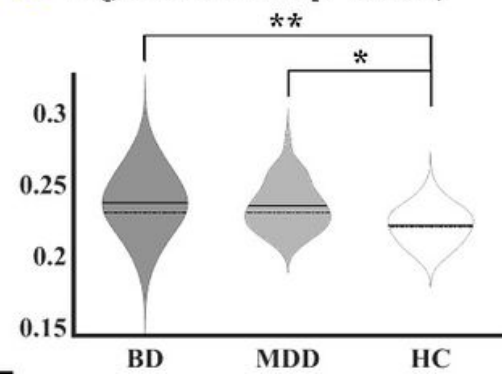

Left Superior frontal $(p<0.001)$

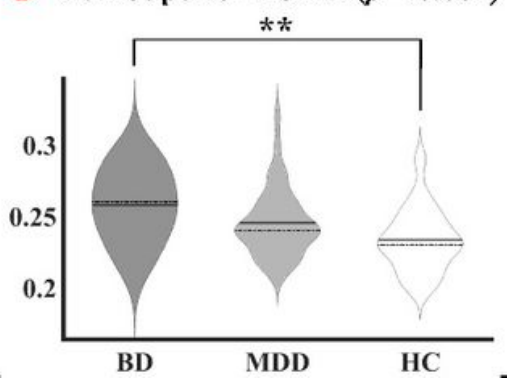

\section{Figure 1}

Violin plots of significant nodal clustering coefficients in high beta band for bipolar disorder, major depressive disorder, and healthy controls. CC, clustering coefficient; BD, bipolar disorder; MDD, major depressive disorder; $\mathrm{HC}$, healthy control. *: $\mathrm{p}<0.05$, * $: \mathrm{p}<0.01$. 
High beta band nodal-level CCs

Left lsthmus cingulate $(p<0.001)$

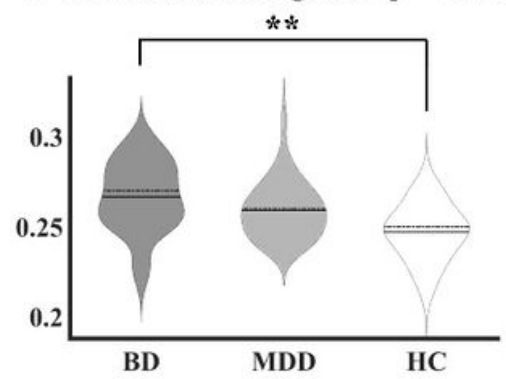

Left Paracentral $(p<0.001)$

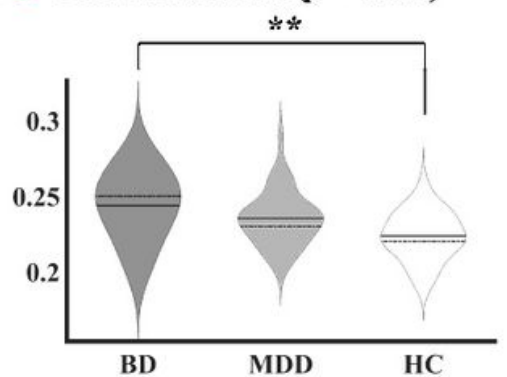

Right Paracentral $(p<0.001)$

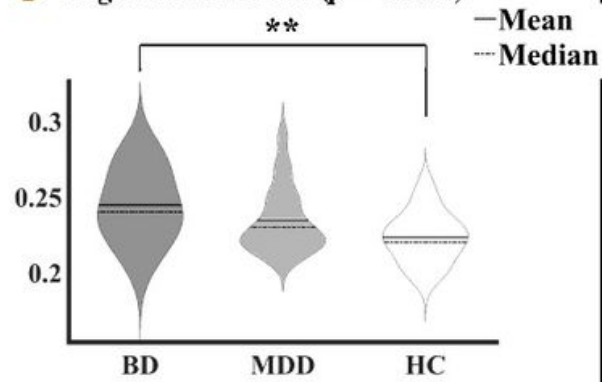

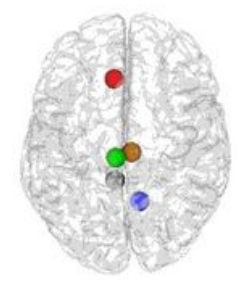

Right Precuneus $(p<0.001)$

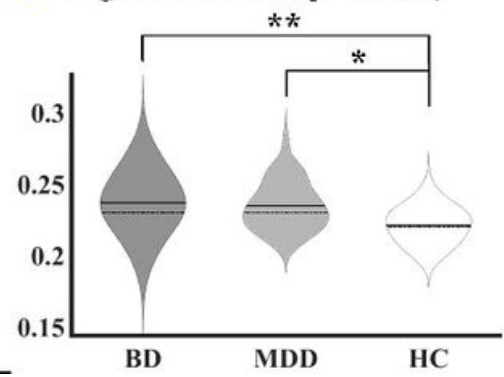

Left Superior frontal $(p<0.001)$

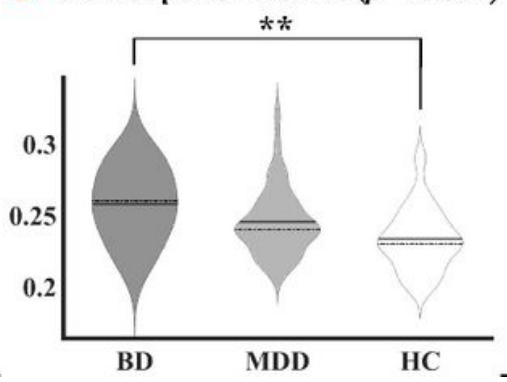

\section{Figure 1}

Violin plots of significant nodal clustering coefficients in high beta band for bipolar disorder, major depressive disorder, and healthy controls. CC, clustering coefficient; BD, bipolar disorder; MDD, major depressive disorder; $\mathrm{HC}$, healthy control. *: $\mathrm{p}<0.05$, **: $\mathrm{p}<0.01$.

BD
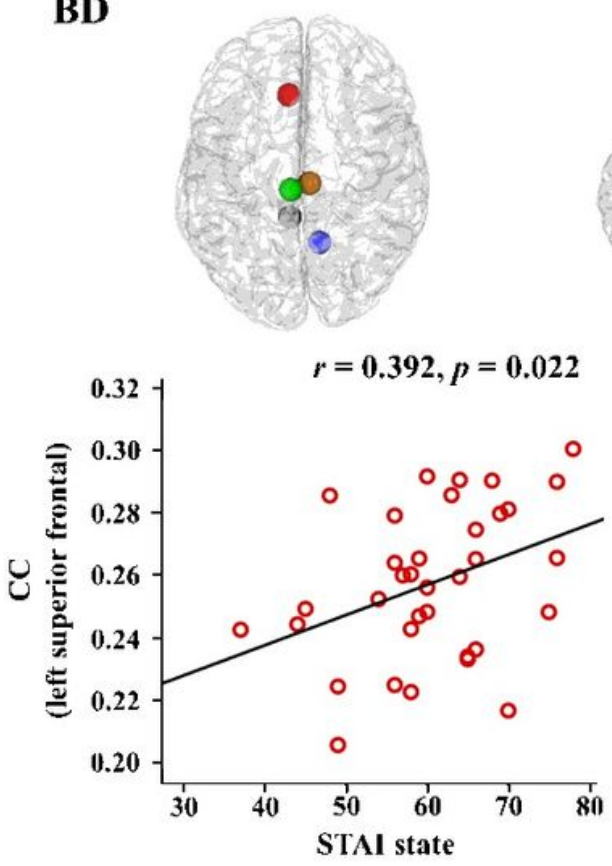
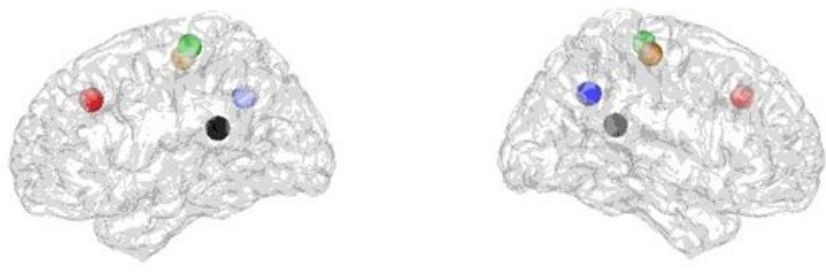

Superior frontal

Paracentral

Paracentral

Isthmus cingulate

Precuneus
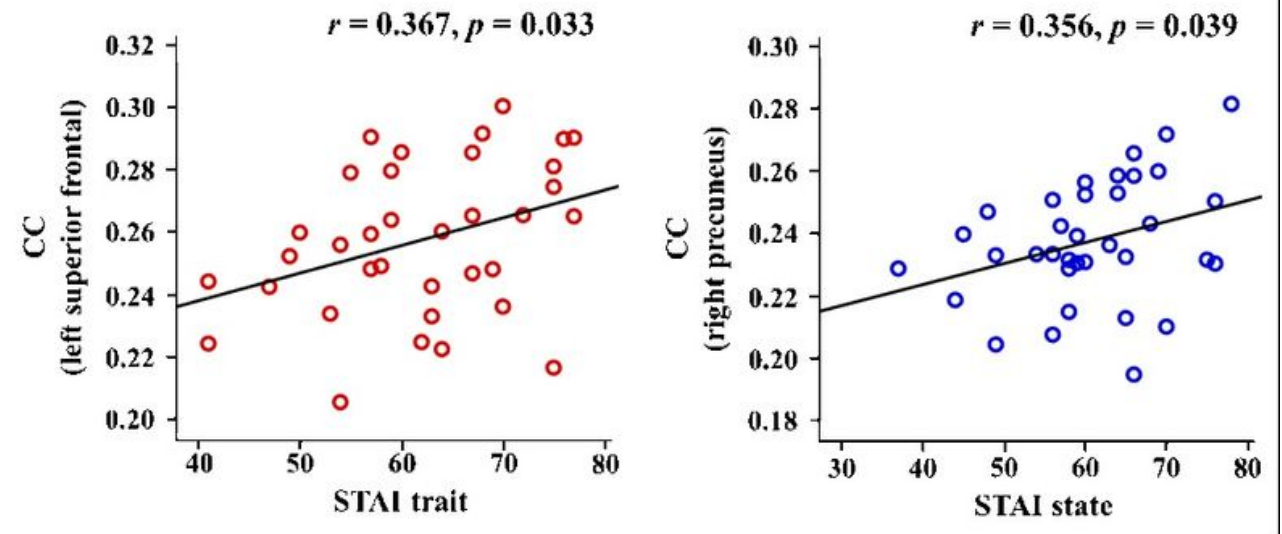
Figure 2

Correlations between nodal clustering coefficients (CC) and psychological measures in high beta band for BD patient group. BD, bipolar disorder; STAI, State-Trait Anxiety Inventory.

BD
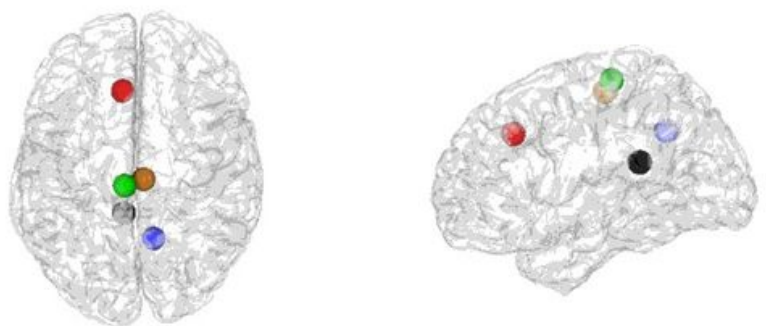

$r=0.392, p=0.022$

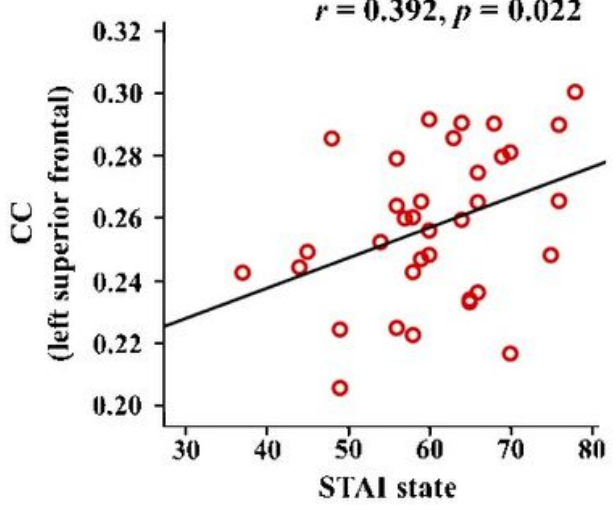

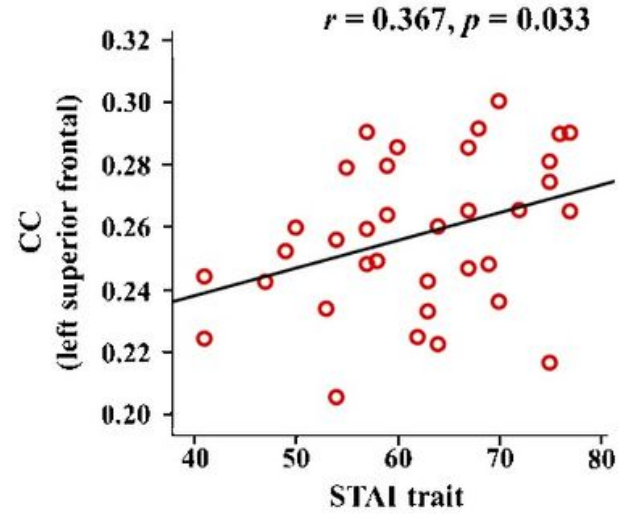

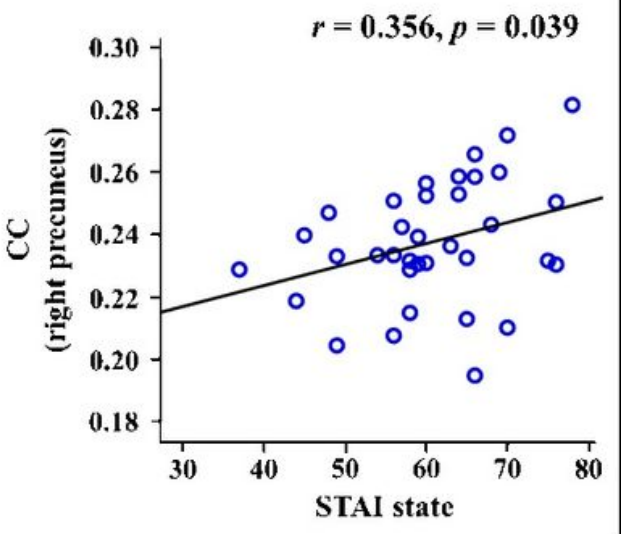

Figure 2

Correlations between nodal clustering coefficients (CC) and psychological measures in high beta band for BD patient group. BD, bipolar disorder; STAI, State-Trait Anxiety Inventory. 